\title{
Synthesis and Detecting Cancer Studies of Up-Conversion Luminescent Materials
}

\author{
Tao LUAN ${ }^{1}$,De-ming HAN ${ }^{1}$, Bai-tao ZENG ${ }^{1}$ and Li-hui ZHAO ${ }^{1 *}$ \\ ${ }^{1}$ College of life science and technology, the Changchun University of Science and \\ Technology, Changchun, China \\ *Corresponding author: zhaolh@cust.edu.cn
}

Keywords: Carcinoma, Up-conversion Luminescent Materials, Diagnosis, Surface Modification.

\begin{abstract}
In this article, designed and preparated rare earth luminescence nanoparticle through different core-shell and doping ratio, improving synthetic conditions, it has uniform size and good crystallinity. Modified nanoparticles with DTT, surface of the nanoparticles with sulphur and combine with oral cancer cell antibodies, under $980 \mathrm{~nm}$ infrared laser excitation, through infrared spectrum detected carcinoma antigen in serum, So as to achieve detection diagnosis to patients with oral drench carcinoma.
\end{abstract}

\section{Introduction}

With the development of science and technology, people pay close attention to nanotechnology. Since the first international conference on nano science and technology, it will be formally nanomaterials science as a new branch of material science, Nanometer materials caused the material world and physics science workers great interest and broad attention and got rapid development[1,2].Due to the unique physical and chemical properties of nanomaterials, make itself has small size effect, macroscopic quantum tunnel effect, surface and interface effect, etc., and make it having a broad prospect in optics, electricity, magnetism, catalysis, sensors biological medicine.

On conversion of nanometer material is a kind of special rare-earth doped inorganic luminescent material, it can be nearly infrared multiphoton mechanism into shortwave radiation, emit ultraviolet or visible light, the anti-Stokes light. This kind of material chemistry stability is good, the luminescence process is hardly affected by temperature, humidity, $\mathrm{pH}$, etc; Up-conversion on the luminescent material is anti - Stokes emitters, the excitation wavelength is generally near infrared or infrared light, and in biological systems, most of the distractors't flourish, reduces the detection background, makes the sensitivity obtained greatly improved; Transformation on the near infrared luminescent material or infrared excitation light long, low energy, so it can be very good to penetrate some biological tissue without damage[3,4].Obversely from the above advantages on conversion nanometer material is a kind of very favorable biomarkers.

At present, the transformation materials are applied in many fields, by doping some material on the surface, such as metal ions, compounds or certain groups, etc., make its can combine with biomedical materials, its chemical properties relative stability, under the near-infrared light stimulate, emit visible light, can be well avoid excitation light damage to the organization, and the application of near infrared excitation light increases the photons in the organization through the limit, the imaging of quantum dots and deep in the animal tissue ability is strong, skin tissue from a green fluorescent 
quantum dots was very weak, and the PEI cladding NaYF4: Yb[5], ER nano materials for $10 \mathrm{~mm}$ in depth within the organization clearly visible fluorescence.

In this paper, based on rare-earth Up-conversion luminescence materials performance is good, little side effects on organisms, High accuracy and high sensitivity to early detection of oral cancer[6].The preparation of Up-conversion luminescent nano materials, modifying the luminescent nanoparticles, and combined with ral cancer specific antibody, make luminescent nanoparticles targeting, specificity and sensitivity, for detecting the oral cancer, and provide certain theoretical basis for comprehensive treatment of oral cancer.

\section{Experimental Materials and Methods}

\section{Laboratory Reagents}

Four fluorine sodium yttrium $\left(\mathrm{NaYF}_{4}\right)$ nanoparticles (purity $\left.99.999 \%\right)$ and cyclohexane solution, hydrochloric acid solution, acetone, deionized water, dithiothreitol (DTT) powder, oral cancer antibody, Tris $\mathrm{HCl}$ buffer.

\section{Experimental Methods}

Up-conversion Luminescence Materials Preparation. Using the sol-gel methods[7], under low temperature, The sol was formed by the hydrolysis and polymerization of the solution, and then generate a certain space structure of the gel, and then processed or decompression drying.

\section{Construction of Transformation on Luminescent Material Surface Amino}

Polyacrylamide (PAAM) of surface ligands, the nanoparticle surface first with hydrochloric acid for the removal of high temperature thermal decomposition and synthesis of surface ligand oleic acid, then polyacrylamide (PAAM) modified, the conversion nanoparticles were successfully dispersed in aqueous solution. The conversion nanoparticles surface modified a lot for bio functionalized amino[9].

\section{Modified Thiol in Antibody Surface Using DTT}

In the Fc fragment, the activity of the antibody was activated by DTT, and the disulfide bind integrate two heavy chain in their hinge area, which can selective adhere and form two parts of the antibody in each part. There is a mercapto group and antigen binding site. then Testing the reducibility of detection antibody by doing SDS-PAGE expriment.

\section{Combination of Up-conversion Luminescence with Antibodies and Detection}

The combination of the amine of luminescence materials and the sulphur of antibody. making the nanostructured luminescent materials and antibody coupling, and the centrifugal, finally wash not combined with antibodies, leaving antibodies and material combined with suspension.

But it doesn't clearly whether finish the combination, only through spectroscopy method on conversion between luminescent material and antibody protein coupling.

\section{Composite Antibodies and Oral Cancer Cell Co-culture}

Using the above methods, we combined nanometer materials with drench cancer antibody, under $37{ }^{\circ} \mathrm{C}$ the nano probe and oral cancer cell co-culture 15 to $30 \mathrm{~min}$, centrifugal, remove the material not combined with the probe, and then the fluorescence were observed under inverted microscope. Compared with the normal 
near-infrared light and $980 \mathrm{~nm}$ under different horizons. It is observation to find out the cancer cells are fluoresce.

\section{Result Analysis}

\section{Purity Test by XRD of Rare-Earth Conversion Luminescence Materials}

The material that was used by the sol-gel method converts,must determine whether its purity meets the requirements, that is the strength of the luminous value can achieve what we hope. So using X ray diffraction (X - ray diffraction), put on the material of lattice plane spacing and diffraction intensity compared with the standard phase diffraction data, determine the material in phase, and then according to the intensity of the diffraction pattern, determine the transformation on rare earth luminescence materials of each phase in the content, so as to determine the purity (as shown in figure $3.1)$.

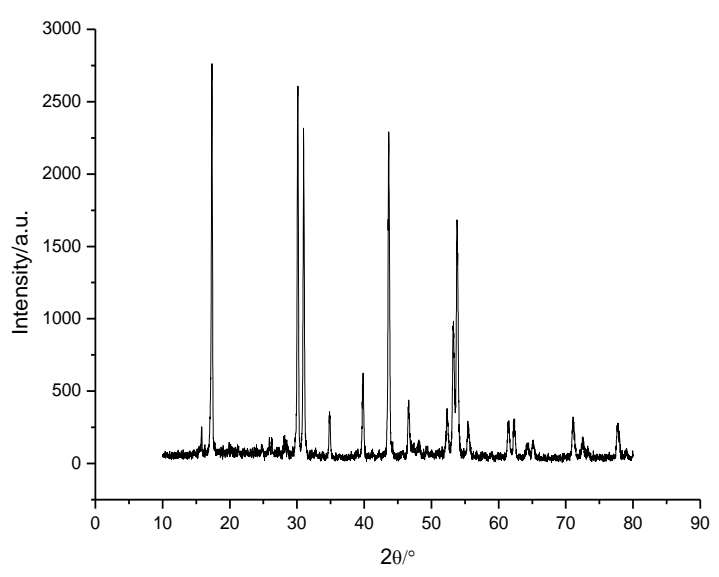

Figure 3.1 X - ray diffraction test purity diagram

\section{On the Rare Earth Conversion Luminescent Material Surface Amino Build Testing Experiment}

In oil phase for preparation of $\mathrm{NaYF}_{4}$ : $\mathrm{Yb}, \mathrm{Er}$ nanoparticles spectrum, $1467 \mathrm{~cm}-1$ derived from the shear type deformation vibration of $-\mathrm{CH} 2$ groups, $1566 \mathrm{~cm}-1$ from in the asymmetric stretching vibrations of the COO-, and $2854 \mathrm{~cm}-1$ and $2925 \mathrm{~cm}-1$ from the $\mathrm{C}-\mathrm{H}$ stretching vibration; in the sample after utilizing polyacrylamide (PAAM) phase transfer, the appearance of absorption peaks of $1157 \mathrm{~cm}-1$ from C-H stretching vibration and $1659 \mathrm{~cm}-1$ and $1572 \mathrm{~cm}-1$ from $\mathrm{N}-\mathrm{H}$ deformation vibration corroborate that about the amine modification of PAAM ligands on the up conversion nanoparticles is successful (Figure 3.2). 


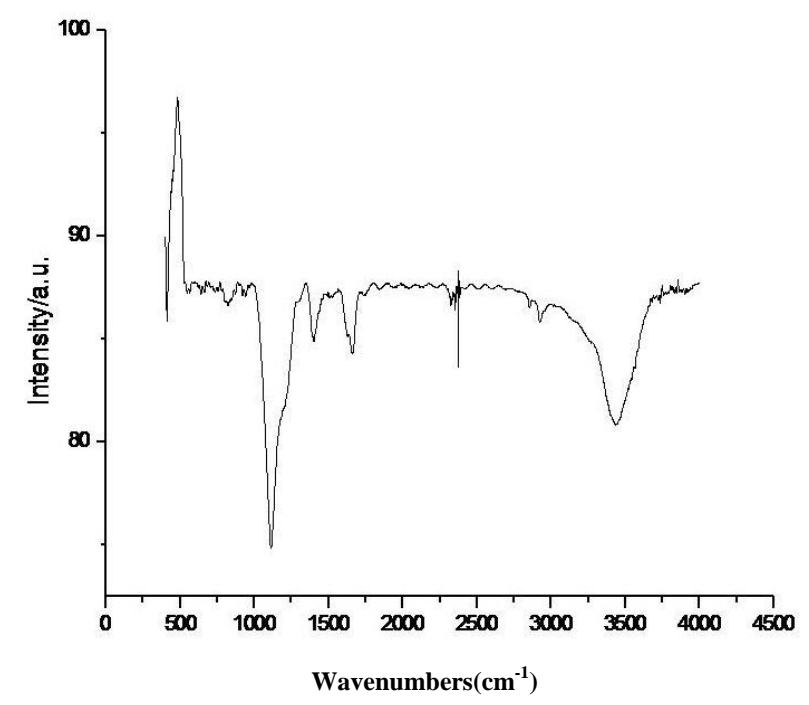

Figure 3.2 Material purity XRD diagram

\section{Surface Detection Antibody Mercapto Modification Experiments}

SDS-PAGE, protein gel electrophoresis, is to make the protein molecules with a large amount of negative charge, neutralize the charge of the protein molecule itself, and eliminate the charge effect of the protein molecule by SDS. After electrophoresis After electrophoresis, utilizing Coomassie brilliant blue to stain , because Kaumas can reach micrograms level, high sensitivity.

Due to the samples were processed with the reducing agent disulfide threitol (DTT), and disconnect the sample protein disulfide bonds, so that electrophoresis sample, our antibody, will be completely reduced to the form of monomer, while one without samples will maintain the dimer form, then it is going to be in the position of the treated samples of 2 times or 3 times the molecular weight. We use the DTT treatment of different concentrations of serum antibody, from right to left, followed by not adding DTT and DTT diluted 1 4 times the results. (Figure 3.3)
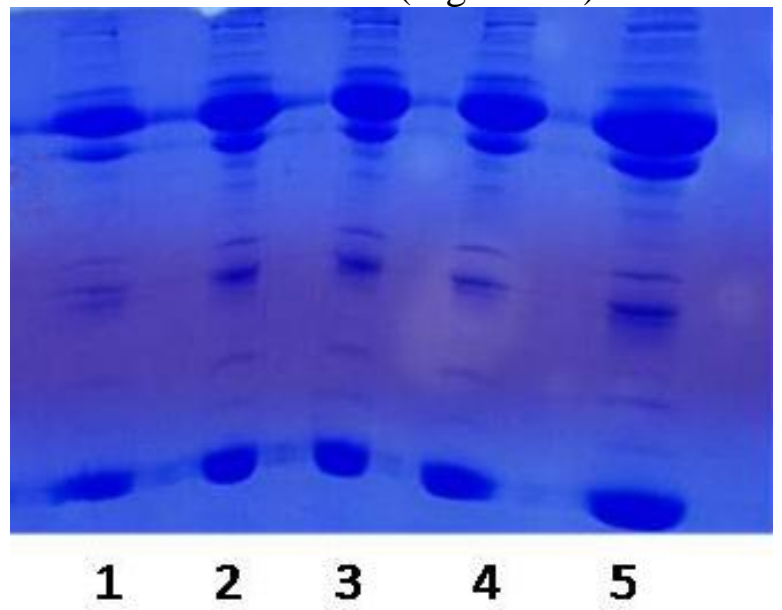

Figure 3.3 SDS-PAGE detection antibody surface modification of thiol

The results of the experiment showed that the concentration of DTT which followed by not adding DTT and DTT diluted 1 4 times from right to left the results was different in the treatment of protein, so the modification of DTT was successful. 


\section{Modification of Thiol Antibodies and Build Amino Nanomaterials Coupling Testing Experiment}

Incubated the nomo materials built by amido with the mercap to modification of antibodies, so as to make the nanostructured luminescent materials and antibody coupling, centrifugal, remove not combined with antibodies, leaving antibodies and material combined with suspension. Using enzyme-linked immunosorbent (ELISA) experiment using antibodies react with two of the specific resistance will be connected to the enzyme object under test, and then through the enzyme and substrate to produce color reaction, used for quantitative determination.

We make Enzyme Linked Immunosorbent (ELISA) kit to detect antibody, the result of the experiment shows positive, explain with antibodies in suspension, and the material and the combination of antibody were washed away, so only in combination with the antibody of material, so the positive results show that combining a success.

Twice by enzyme-linked immunosorbent assay is positive, we have to material and antibodies have been combined. Can be used for cancer detection, into the next phase of the experiment.

\section{Oral Cancer Cells and Antibodies Composites Trained}

Using the above method we will nano materials combined with squamous carcinoma of antibody, in $37{ }^{\circ} \mathrm{C}$ under the nanometer probe with squamous cancer cells develop 15 to $30 \mathrm{~min}$, centrifugal, remove not combined with the probe, and then the fluorescence were observed under inverted microscope. Compared with normal near-infrared light and $980 \mathrm{~nm}$ under different horizons, observe whether the observed fluoresce K562 cells. If all can see that on the resulting conversion luminescence materials fluorescent probes can be used to completely cell markers.

\section{Discussion}

The early diagnosis of cancer is a diagnostic and treatment method which aims at the early patients of cancer. Its goal is early detection and treatment to relieve the patients' sufferings and the burdens of spirit and economy. The early patients of cancer can get well soon through the early diagnosis of cancer. The nanotechnology is possibly one of the most promising means of technology for diagnosing and preventing cancer. But now, the application of nanotechnology is still at an incomplete stage. Only a few drugs are used in the clinic. What we solve, first and foremost, are the security issues of this material for organisms. It is a key issue we all care about. The nanotechnology has different impacts on the organisms' organs, tissues and cells, small to the molecule, genes and other different levels.

\section{References}

[1] P. J. Cregg, K. Murphy, A. Mardinoglu. Inclusion of interactions in mathematical modelling of implant assisted magnetic drug targeting.Applied Mathematical Modelling.36 (2012) 1-34.

[2]Q. Liu, Y. Sun, T. S. Yang,W.Feng,C.G.Li,F.Y.Li. Sub10 nm Hexagonal lanth anidedoped NaLuF4upconversion nanocrystals for sensitive bioimaging in vivo. J Am Chem Soc.133(2011) 1722-1712. 
[3] K. Song, X. G. Kong, X. M. Liu. Aptamer optical biosensor without bio-breakage using upconversion nanoparticles as donors. Chem Commun. 48 (2012) 1156-1158.

[4] Amitava Patra, Christopher S. Friend, Rakesh Kapoor, Paras N. Prasad. Upconversion in Er3+:ZrO2; NaYF4:Yb Nanocrystals. J. Phys. Chem.106 (2002)1909-1912.

[5] Jiang S, Zhang Y. Upconversion Nanoparticles-Based FRET System for Study of SiRNA in Live Cells.Langmuir.26(2010)6689-6694.

[6] Xia Lu.targeted on conversion nano photosensitizer and medicine application research; [Ph.D. Thesis] changchun university of science and technology, 2012 .

[7] V. R. N. Fiorenzo, M. Venkataramanan, G. Christopher et al. The Active-Core/Active-Shell Approach: A Strategy to Enhance the Upconversion Luminescence in Lanthanide-Doped Nanoparticles..Advanced Functional Materials. 19(2009) 2924-2929.

[8]K. Song, X. G. Kong, X. M. Liu, et al. Aptamer optical biosensor without biobreakage using upconversion nanoparticles as donors. Chem Commun.48(2012) 1156-1158.

[9] P. Huang, W. Zheng, S.Y. Zhou, Zhou,D.T. Tu,Z. Chen,H.M. Zhu et al. LanthanideUpconversion Nanoprobes for the Detection of Disease Biomark ers. Angew. Chem. Int. Ed. 53(2014)1252-1257. 